\title{
ANÁLISIS DE LA INVESTIGACIÓN "IMAGINARIOS SOCIALES DEL DESARROLLO HUMANO, SUBYACENTES EN LAS POLÍTICAS DE RESOCIALIZACIÓN DE MUJERES PROFESIONALES INTERNAS, EN EL CENTRO PENITENCIARIO VILLA CRISTINA DE LA CIUDAD DE ARMENIA, QUINDÍO, COLOMBIA" REALIZADA POR QUICENO, J; MORALES, L \& CUELLAR, L, 2015.
}

\author{
ANALYSIS OF THE RESEARCH "SOCIAL IMAGINARIES OF HUMAN \\ DEVELOPMENT, UNDERLYING THE POLICIES OF RESOCIALIZATION OF \\ INTERNAL PROFESSIONAL WOMEN, IN THE VILLA CRISTINA PENITENTIARY \\ CENTER IN THE CITY OF ARMENIA, QUINDÍO, COLOMBIA" CONDUCTED BY \\ QUICENO, J; MORALES, L \& CUELLAR, L, 2015.
}

\author{
Martha Cecilia Rambal-Simanca ${ }^{1} *$ (D); Leylis Acuña-Sauriht ${ }^{2}$. \\ 1.: Universidad del Norte, Atlántico, Barranquilla, Colombia.mrambal@uninorte.edu.co \\ 2. Universidad del Norte, Atlántico, Barranquilla, Colombia. 1sauriht@uninorte.edu.co \\ *Correspondencia del Autor: Martha Cecilia Rambal-Simanca, correo electrónico: mrambal@uninorte.edu.co.
}

\section{RESUMEN}

El presente artículo es una carta al editor, en el se realiza un análisis a la investigación realizada por Quiceno, J; Morales, L \& Cuellar, L (2015) denominada "Imaginarios sociales del desarrollo humano, subyacentes en las políticas de resocialización de mujeres profesionales internas, en el centro penitenciario Villa Cristina, de la ciudad de Armenia, Quindío, Colombia. Esta es una investigación cualitativa desde el método fenomenológico, utilizó técnicas para la recolección de la información como la entrevista y el grupo focal; participaron mujeres reclusas que son profesionales, por lo que el muestreo es no probabilístico de conveniencia. Se analizaron los datos en coherencia con el método fenomenológico, concluyendo que las políticas de resocialización aplicadas en ese centro carcelario femenino no logran cumplir con su objetivo, ni se emplean apropiadamente, por ello, los autores proponen el diseño de una política pública de resocialización diferencial.

Los criterios utilizados para evaluar esta investigación fueron: los propósitos del estudio, la revisión de la literatura, el diseño del estudio, el muestreo, la recopilación datos, el análisis de datos, el rigor general, las conclusiones y la trascendencia. A partir de lo anterior, se puede afirmar que la investigación de Quiceno y otros (2015) cumple con todos los criterios que evidencian el rigor teórico y metodológico empleado, por lo cual, goza de validez y confiabilidad.

Palabras clave: imaginarios sociales; desarrollo humano; políticas de resocialización; sistema penitenciario.

\section{Cómo citar:}

Rambal-Simanca, Martha Cecilia; Acuña-Sauriht, Leylis. (2021). Análisis de la investigación "Imaginarios sociales del desarrollo humano, subyacentes en las políticas de resocialización de mujeres profesionales internas, en el centro penitenciario Villa Cristina de la ciudad de Armenia, Quindío, Colombia” realizada por Quiceno, J; Morales, L \& Cuellar, L, 2015. Revista de Investigaciones Universidad del Quindio, 33(1), 126-131. https://doi.org/10.33975/riuq.vol33n1.321 


\section{ABSTRACT}

This paper is a letter to the editor, in which an analysis of the research carried out by Quiceno, J; Morales, L \& Cuellar, L (2015) is made, called "Social Imaginaries of Human Development, underlying in the Policies of Resocialization of Internal Professional Women, at Villa Cristina Penitentiary, in the city of Armenia, Quindío, Colombia. This is a qualitative research from the phenomenological method, used techniques for the collection of information such as the interview and the focus group; female prisoners who are professionals participated, so the sampling is non-probabilistic of convenience. The data were analyzed in coherence with the phenomenological method, concluding that the resocialization policies applied in this female prison center do not achieve their objective, nor are they used properly, therefore, the authors propose the design of a public policy of differential resocialization.

The criteria used to evaluate this research were: study purposes, literature review, study design, sampling, data collection, data analysis, general rigor, conclusions, and significance. Based on the above, it can be affirmed that the research by Quiceno et al. (2015) meets all the criteria that show the theoretical and methodological rigor used, therefore, it has validity and reliability.

Keywords: social imaginary; human development; resocialization policies; prison system.

\section{CARTA AL EDITOR}

La presente carta al editor desarrolla el análisis del articulo denominado "Imaginarios sociales del desarrollo humano, subyacentes en las políticas de resocialización de mujeres profesionales internas, en el centro penitenciario Villa Cristina, de la ciudad de Armenia, Quindío, Colombia", realizada por Quiceno, J; Morales, L \& Cuellar, L (2015), cuyos resultados fueron publicados en la revista de la Universidad de Quindío, Colombia volumen 27(1), p. 9-20.

Los criterios para evaluar, fueron los propuestos por el formulario de revisión crítica - cualitativa de Letts et al, 2007, que incluyen criterios de evaluación como: recopilación datos, análisis de datos, el muestreo, propósitos del estudio, revisión de la literatura, diseño del estudio, rigor general, conclusiones y trascendencia; y los otros criterios fueron tomados del texto denominado "Pautas para la revisión crítica de estudios cualitativos, basado en las pautas para los estudios cualitativos del formulario de revisión crítica" de Law, M., Stewart, D., Letts, L., Pollock, N., Bosch, J. y Westmorland, M., 1998. Este análisis es complementado con fundamentos realizadas a partir de las referencias bibliográficas y notas de clase de la asignatura denominada Análisis de Datos Cualitativos desarrollada en el Doctorado en Ciencias Sociales de la Universidad del Norte en Barranquilla, Colombia.

Quiceno y otros (2015) hacen un recorrido por el concepto de imaginario social de Cornelius Castoriadis (1980), pasando por la teoría de desarrollo a escala humana de Max-Neef (1993) hasta explicar el razonamiento sobre el aparato disciplinario desde Foucault (2002), evidenciando la aplicabilidad entre estas teorías y el contexto penitenciario de las internas de la cárcel de mujeres Villa Cristina, en la ciudad de Armenia, Quindío.

El fundamento de la investigación cualitativa, es tener claro los propósitos del estudio, pues, estos 
son los que orientan al investigador junto con el marco de referencia conceptual, al tiempo que le permitirán, seguir indagado a profundidad hasta lograr la pertinencia y relevancia de su estudio (Sandoval, 2002; Maxwell, 2008), para la investigación de Quiceno, et, al, 2015, el método elegido fue el fenomenológico, tal como lo plantea Law, et, al. (1998),

Phenomenology answers the question: "What is it like to have a certain experience?" It seeks to understand the phenomenon of a lived experience - this may be related to an emotion, such as loneliness or depression, to a relationship, or to being part of an organization or group (p.2).

Por lo anterior, puede deducirse que el método fenomenológico elegido por Quiceno, et, al (2015) es coherente con el propósito del estudio, que es comprender el imaginario de sociedad a partir de la experiencia de las reclusas y las teorías que lo fundamentan, como lo son: el desarrollo a escala humana de Max-Neef (1993), el concepto de imaginario social de Cornelius Castoriadis (1980) y el razonamiento del aparato disciplinario de Foucault (2002).

Las técnicas utilizadas fueron la entrevista y el grupo focal, elegidas de manera apropiada por los investigadores, ya que son herramientas cualitativas de recolección de información. La entrevista, permite acercarse al objeto de estudio de manera directa, permitiendo que cada entrevistado, "exprese libremente en forma detallada sus motivaciones, creencias, y sentimientos sobre un tema" Mejía (como se citó en Scribano, 2007); y el grupo focal, por su parte, permite que el grupo de participantes pueda reflexionar sobre el objeto de estudio, permitiéndole al investigador conocer los consensos, los disensos e ir validando sus conclusiones (Sandoval, 2020).

Los investigadores afirman que la población participante en el estudio, son mujeres profesionales internas en la Cárcel Villa Cristina de la ciudad de Armenia, por lo que se infiere se utilizó un muestreo no probabilístico de conveniencia (Lynch, s.f.), ya que la selección de las participantes fue intencional basada en los propósitos del estudio. No obstante, los investigadores no explicitan el número de personas entrevistadas, ni cuantas fueron las participantes del grupo focal, así mismo, se desconoce si existe algún impedimento de confidencialidad al respecto, ya que los autores tampoco lo evidencian en su artículo.

La investigación analizó los datos en coherencia con el método fenomenológico, “explorando significados mediante las experiencias narradas y documentas, para lo cual se procesó una matriz de datos codificados" (Quiceno, et, al, 2015, p. 12), dentro de los hallazgos están: desde Max - Neef, las políticas de resocialización de la cárcel Villa Cristina de la ciudad de Armenia, limita el desarrollo humano de las mujeres reclusas que son profesionales, por cuanto, el sistema de resocialización no satisface las necesidades de las participantes; desde Cornelius Castoriadis el imaginario instituido del proceso de resocialización es deficiente, ya que no tiene en cuenta "a la persona y su potencial humano" (Quiceno, et, al, 2015, p. 16) y desde Foucault, las críticas al proceso se centran en los medios empleados para conseguirlo donde "el cuerpo sólo se convierte en fuerza útil cuando es a la vez cuerpo productivo y cuerpo sometido", generándose un "suplicio simbólico" (Quiceno, et, al, 2015, p. 18)

La investigación denota cientificidad, evidenciándose cuatro componentes fundamentales, 1) la credibilidad en la solidez teórica y metodológica, 2) la transferibilidad se logró per se, puesto que es una característica de los estudios cualitativos, en cuanto a 3) la fiabilidad y 4) la confiabilidad de la investigación, es garantizada a través del proceso de arbitraje realizado por editores de la revista, 
Análisis de la investigación "Imaginarios sociales del desarrollo humano, subyacentes en las políticas de resocialización de mujeres profesionales internas, en el centro penitenciario Villa Cristina de la ciudad de Armenia, Quindío, Colombia" realizada por Quiceno, J; Morales, L \& Cuellar, L, 2015.

así como también, fue evaluada por las autoridades académicas de la Universidad del Quindío en el marco del programa de Maestría que cursaban los investigadores.

Por otra parte, en cuanto a las conclusiones de la investigación, se puede afirmar que fueron pertinentes con relación a los hallazgos del estudio, dentro de ellas se destacan, que las políticas de resocialización aplicadas en ese centro carcelario femenino no lograr cumplir con su objetivo, ni se emplean apropiadamente, debido al hacinamiento, a la no participación de la población de mujeres reclusas dentro de su diseño y a la incoherencia que se presenta entre los expresado en la normatividad y su aplicación, por ello, los autores plantean que se requiere de una política pública diferencial que incluya un manual de convivencia y una formación encaminada al desarrollo humano, que se reoriente al reconocimiento de las habilidades y capacidades de las mujeres internas, en especial, de las que son profesionales y pueden aportar a distintos procesos dentro del centro carcelario (Quiceno, et, al, 2015). Todos estos hallazgos, retroalimentan la práctica social en el contexto penitenciario femenino y enriquecen el acervo teórico en materia de desarrollo humano.

A partir de todo lo anterior, se concluye que la investigación cualitativa de Quiceno y otros (2015) cumple con todos los criterios que evidencian el rigor teórico y metodológico empleado, por lo cual, goza de validez y confiabilidad científica.

\section{REFERENCIAS}

1. Castoriadis, C. (1980). El mito del desarrollo. Reflexiones sobre el desarrollo y la racionalidad. Buenos Aires. Edit. Kairós. Disponible en: http://books.google.com.co/books?id=DO5J6S5BwE $\mathrm{wC} \&$ printsec $=$ frontcover\&hl $=$ es\&source $=$ gbs_ge_summary_r\&cad $=0 \# \mathrm{v}=$ onepage $\& \mathrm{q} \& \mathrm{f}=$ false.

2. Foucault, M. (2002). Vigilar y castigar: nacimiento de la prisión. Buenos Aires: Edit. Siglo XXI.

3. Law, M., Stewart, D., Letts, L., Pollock, N., Bosch, J. y Westmorland, M. (1998). Pautas para la revisión crítica de estudios cualitativos, basado en las pautas para los estudios cualitativos del formulario de revisión crítica. Universidad McMaster.

4. Letts, L., Wilkins, S., Law, M., Stewart, D., Bosch, J. y Westmorland, M. (2007). Formulario de revisión crítica - cualitativa. Estudios (versión 2.0) (C) Universidad McMaster.

5. Lynch, G (s.f.). Sampling. The Oxford seminar. Oxford University, Inglaterra, Reino Unido. Disponible en: https://www.kent.ac.uk/religionmethods/documents/Sampling.pdf

6. Max-Neef, M. A. (1993). Desarrollo a escala humana. Conceptos, aplicaciones y algunas reflexiones. Montevideo: Editorial Nordam-Comunidad.

7. Maxwell J.A., (2008). Designing a Qualitative Study. Handbook of applied social research methods (p. 214- 253).

8. Quiceno, J., Morales, L., y Cuellar, L. (2015). Imaginarios sociales del desarrollo humano, subyacentes en las políticas de resocialización de mujeres profesionales internas, en el Centro Penitenciario Villa Cristina, de la ciudad de Armenia, Quindío, Colombia. Revista de Investigaciones Universidad del Quindio, 27(1), pp. 9-20.

9. Sandoval, C. (2020). Grupo focal (Notas de clase). Barranquilla, Colombia. Doctorado en ciencias sociales de la Universidad del Norte.

10. Sandoval, C (2002). La Formulación y Diseño de los Procesos de Investigación Social Cualitativos. Investigación cualitativa (p. 111-128). Bogotá, Colombia: ICFES-ASCUN.

11. Schettini, P \& Cortazzo, I (2015). Análisis de datos cualitativos. Análisis de datos cualitativos en la investigación social procedimientos y herramientas para la interpretación de información cualitativa (p. 28-43). La plata, Argentina: Editorial Universidad de la Plata.

12. Scribano, A. (2007). El proceso de investigación social cualitativo. Buenos Aires, Argentina: Editorial Prometeo. 
ANEXO:

Cuadro 1. Análisis crítico de la investigación de Quinceno, et al, 2015.

\begin{tabular}{|c|c|}
\hline CRITERIOS & PREGUNTAS \\
\hline Propósito del estudio. & $\begin{array}{l}\text { ¿Fue el propósito y / o investigación / Pregunta formulada claramente? } \\
\text { o sí (X) } \\
\text { o no () }\end{array}$ \\
\hline Literatura. & $\begin{array}{l}\text { ¿Fueron relevantes los antecedentes de la literatura revisada? } \\
\text { o sí (X) } \\
\circ \text { no() }\end{array}$ \\
\hline Diseño del estudio. & $\begin{array}{l}\text { ¿Cuál fue el diseño? } \\
\text { ○ Fenomenología }(\mathrm{X}) \\
\text { ○ Etnografía } \\
\text { ○ Teoría fundamentada } \\
\text { ○ Investigación de acción participativa } \\
\text { ○ Otro } \\
\text { ¿Cuál fue la perspectiva teórica identificada? } \\
\text { O sí }(\mathrm{X}) \\
\text { ○ no } \\
\text { Método (s) utilizado (s): } \\
\text { o observación participante } \\
\text { o entrevistas (X) } \\
\text { O revisión de documentos } \\
\text { o grupos focales }(\mathrm{X}) \\
\text { ○ otro }\end{array}$ \\
\hline Muestreo. & $\begin{array}{l}\text { ¿Fue descrito el proceso de selección? } \\
\text { o sí }(\mathrm{X}) \\
\text { o no } \\
\text { ¿Se realizó el muestreo hasta que se alcanzó la saturación en los datos? } \\
\text { o sí }(\mathrm{X}) \\
\text { o no } \\
\text { ¿Se obtuvo el consentimiento informado? } \\
\text { O sí } \\
\text { O no } \\
\text { o no abordado }(\mathrm{X})\end{array}$ \\
\hline Recopilación de datos. & $\begin{array}{l}\text { Claridad descriptiva: } \\
\text { Descripción clara y completa de: } \\
\text { Sitio: ○ sí ○ no (X) } \\
\text { Participantes: ○ sí ○ no }(\mathrm{X}) \\
\text { Rol de investigador y relación } \\
\text { con los participantes: } \\
\text { ○ sí ○ no (X) } \\
\text { Identificación de supuestos y } \\
\text { sesgos del investigador: } \\
\text { o sí ○ no (X) } \\
\text { Rigor procesal } \\
\text { ¿Se utilizó rigor procesal en los datos estrategias de recolección? } \\
\text { ○ sí } \\
\text { o no } \\
\text { o no abordado (X) }\end{array}$ \\
\hline
\end{tabular}


Análisis de la investigación "Imaginarios sociales del desarrollo humano, subyacentes en las políticas de resocialización de mujeres profesionales internas, en el centro penitenciario Villa Cristina de la ciudad de Armenia, Quindío, Colombia” realizada por Quiceno, J; Morales, L \& Cuellar, L, 2015.

\begin{tabular}{|c|c|}
\hline Análisis de los datos. & $\begin{array}{l}\text { Rigor analítico } \\
\text { ¿Los análisis de datos fueron inductivos? } \\
\circ \text { sí }(\mathrm{X}) \circ \text { no } \circ \text { no abordado } \\
\text { ¿Los resultados fueron consistentes con los datos?, ¿Refleja los datos? } \\
\circ \text { sí }(\mathrm{X}) \circ \text { no } \\
\text { Auditabilidad } \\
\text { ¿Muestran el sendero de decisión desarrollado? } \\
\circ \text { sí } \odot \text { no } \circ \text { no abordado }(\mathrm{X}) \\
\text { ¿El proceso de análisis de los datos fue descrito adecuadamente? } \\
\circ \text { sí }(\mathrm{X}) \circ \text { no } \circ \text { no abordado } \\
\text { Conexiones teóricas } \\
\text { ¿Hice una imagen significativa de la fenómeno en estudio emerge? } \\
\circ \text { sí }(\mathrm{X}) \\
\circ \text { no }\end{array}$ \\
\hline Rigor general. & $\begin{array}{l}\text { ¿Había evidencia de los cuatro componentes de confiabilidad? } \\
\text { Credibilidad } \circ \text { sí }(\mathrm{X}) \circ \text { no } \\
\text { Transferibilidad } \circ \text { sí }(\mathrm{X}) \circ \text { no } \\
\text { Fiabilidad } \circ \text { sí }(\mathrm{X}) \circ \text { no } \\
\text { Confiabilidad } \circ \text { sí }(\mathrm{X}) \circ \text { no }\end{array}$ \\
\hline Conclusiones en trascendencia. & $\begin{array}{l}\text { ¿Las conclusiones fueron apropiadas dados los hallazgos del estudio? } \\
\circ \text { sí }(\mathrm{X}) \circ \text { no } \\
\text { ¿Los hallazgos contribuyeron al desarrollo de la teoría y futuro de la práctica } / \\
\text { investigación? } \\
\text { ○ sí }(\mathrm{X}) \circ \text { no }\end{array}$ \\
\hline
\end{tabular}

Fuente: Formulario de revisión crítica - cualitativa, Estudios (versión 2.0) (C Letts, L., Wilkins, S., Law, M., Stewart, D., Bosch, J. y Westmorland, M., 2007, Universidad McMaster. 